\title{
Mixing Matrix Estimation Algorithm for Underdetermined Instantaneous Mixing Model
}

\author{
Fan Shi ${ }^{\mathrm{a}}{ }^{*}$ and Chuang Liu ${ }^{\mathrm{b}}$ \\ ${ }^{a}$ Faculty of Maritime and Transportation, Ningbo University, Ningbo, 315211, China \\ ${ }^{b}$ College of Information and Communication Engineering, Harbin Engineering University, Harbin, 150001, China
}

\begin{abstract}
Estimating the mixing matrix is a research that focuses on underdetermined blind source separation. In order to get a more accurate estimated mixing matrix, we investigate a novel algorithm for mixing matrix estimation. Firstly, a new method for detecting single source points was introduced. Then, we reckoned the signal quantity and initial clustering centers by adopting an improved clustering method based on the potential function. Finally, the point density theory and the initial clustering centers were utilized to get more accurate clustering centers and estimated the mixing matrix. The simulation results illustrate that we can obtain the more accurate and stable estimation of the mixing matrix by using the proposed algorithm.
\end{abstract}

Keywords: underdetermined blind source separation; mixing matrix estimation; potential function; single source points

(Submitted on November 7, 2018; Revised on December 3, 2018; Accepted on January 3, 2019)

(C) 2019 Totem Publisher, Inc. All rights reserved.

\section{Introduction}

As the mixing channel and the source signals are not established, how to get each source signal from mixed signals has been a worthy problem in science and engineering. Therefore, blind source separation (BSS) is put forward, and more and more researchers have been involved in research corresponding BSS algorithms. The BSS algorithm is now widely used in areas such as image processing [1-2], ship noise processing [3-4], bio-medical engineering [5-6] and wireless system [7-8]. In recent years, new methods are proposed unceasingly, and the theory of BSS is modified and improved. So far, BSS has been classed into standard blind source separation (SBSS), overdetermined blind source separation (OBSS) and underdetermined blind source separation (UBSS). For its extensive applicability, UBSS is now a hot topic in this field. Due to the limit of practical conditions, the number of sensors may be less than the sources. In this situation, how to solve the problem is relevant to the UBSS problem, which is even trickier than the other two problems. One of the most important methods to solve the SBSS problem and the OBSS problem is independent component analysis (ICA) [9-10]. Because the system of the UBSS model is irreversible, ICA is not suitable for solving this problem. Generally, the sparse component analysis (SCA)based two-step method [11-12] is a fairly important method for UBSS problems. The first step of the method is to estimate a mixing matrix, and the next step is source signal recovery. Since an accurate estimation of the mixing matrix is the basis of signals recovery, the accuracy of the first step is very important. Therefore, the purpose of this study was to determine the estimation of the mixing matrix, the sparsity of signals and how to use the sparsity to determine the performance of the SCA methods. As a matter of fact, some signals are sparse in the time domain, but most signals are not. To obtain more sparse features of signals, the method of time-frequency (t-f) transformation is usually adopted because signals can present better sparsity in the t-f domain. Short-time Fourier transform (STFT) is a main t-f transform method for its implementation simplicity. Better sparsity leads to better linear clustering property. Combining this property and clustering algorithms, the mixing matrix is then generated with received signals. However, there will be some outliers if the signal in the t-f plane is not sufficiently sparse or noises exists. To solve the problem of outliers, the methods [13-17] based on single source point detection and single source region detection get more and more attention. Single source regions denote the t-f regions that only a source signal exists or only a source signal is dominant. Similarly, a single source point is denoted as the t-f point that

\footnotetext{
* Corresponding author.

E-mail address: shifan@nbu.edu.cn
} 
only one source signal exists or only one source signal is dominant. These single source points make the observed signals present better linear clustering property. The better the performance of single source points detects, the higher the estimation accuracy is. Many scholars have researched the corresponding algorithms and have done a lot of work on single source region detection and single source point detection. From Literature [18], some t-f regions where only a source signal exists are first chosen by utilizing the $\mathrm{t}-\mathrm{f}$ ratios, then are used to estimate the mixing matrix. Literature [19] extended the algorithm in Literature [18] to solve the problem of the time-delay mixing model. Lu [20] introduced an algorithm based on single source detection to estimate mixing matrix. First, the t-f region of observed signals is divided into many disjoint regions. Then, detect the regions where only a source signal occurs by using the method of eigenvalue decomposition. Finally, obtain the mixing matrix estimation with the hierarchical clustering. All of the above algorithms utilize single source regions. Based on the single source region algorithms, for the underdetermined instantaneous mixing model, scholars propose many single source point algorithms to get the estimation of the mixing matrix. Reju [21] investigated a method to detect the single source points by judging whether the direction of the real and imaginary parts of STFT coefficients of observed signals are the same, and gets the estimated mixing matrix utilizing a hierarchical clustering algorithm. Dong [22] studied an algorithm for estimating the mixing matrix. First of all, the real vector and the imaginary vector of $t-f$ coefficients of observed signals are normalized. Then, the single source points are detected by measuring the distance of the normalized real vector and the normalized imaginary vector. Finally, the robustness clustering algorithm based on similarity is studied to get an estimated mixing matrix. Besides, considering single source points, many algorithms are used to estimate the mixing matrix in the underdetermined time-delay mixing model. The major contributions of this is a mixing matrix method with better performance for UBSS problems is investigated. Through proposing a new single source points detection algorithm and an improved clustering algorithm, the more accurate mixing matrices can be obtained.

In the remainder of this paper. Section 2 introduces the instantaneous mixing model about UBSS problems, and the theory of single source points detection is analyzed. Section 3 presents an improved clustering algorithm. In Section 4 , we carry out several simulations, and the results and correlation analysis are shown. Section 5 gives out the conclusion.

\section{Problem Formulation}

The underdetermined instantaneous mixing model without noise interference can be expressed as

$$
x(t)=A s(t)
$$

where $x(t)=\left[x_{1}(t), x_{2}(t), \cdots, x_{N}(t)\right]^{T}$ denotes the mixed signal vector at the time point $t$. The mixing matrix is represented by $A=\left[a_{1}, a_{2}, \cdots, a_{M}\right] \in R^{N \times M},(M>N)$, in which, $a_{i}$ denotes the mixing matrix's column vector. $s(t)=\left[s_{1}(t), s_{2}(t), \cdots, s_{M}(t)\right]^{T}$ denotes the source signal vector at the time point $t$. The Equation (1) can also be denoted as

$$
x(t)=\sum_{i=1}^{M} a_{i} s_{i}(t)
$$

In order to get better sparsity, STFT is applied in this paper. The STFT of the $n^{\text {th }}$ mixed signal is expressed as

$$
X_{n}(t, f)=\int_{-\infty}^{\infty} x_{n}(\tau) h(t-\tau) e^{-j 2 \pi f \tau} \mathrm{d} \tau
$$

Where $h(t)$ is the window function. The STFT of the $m^{\text {th }}$ source signal can be written as

$$
S_{m}(t, f)=\int_{-\infty}^{\infty} S_{m}(\tau) h(t-\tau) e^{-j 2 \pi f \tau} \mathrm{d} \tau
$$

The $\mathrm{t}$-f representation of the mixed signals is

$$
X(t, f)=A S(t, f)=\sum_{i=1}^{M} a_{i} S_{i}(t, f)
$$

$X(t, f)=\left[X_{1}(t, f), X_{2}(t, f), \cdots, X_{N}(t, f)\right]^{T}$ and $S(t, f)=\left[S_{1}(t, f), S_{2}(t, f), \cdots, S_{M}(t, f)\right]^{T}$ are the mixed signal after STFT and the source signal after STFT, respectively. In this paper, two mixed signals are taken as an example to analyze the 
algorithms of this paper. The Equation (5) can also be written as

$$
\left[\begin{array}{l}
X_{1}(t, f) \\
X_{2}(t, f)
\end{array}\right]=\left[\begin{array}{llll}
a_{11} & a_{12} & \cdots & a_{1 M} \\
a_{21} & a_{22} & \cdots & a_{2 M}
\end{array}\right]\left[\begin{array}{c}
S_{1}(t, f) \\
S_{2}(t, f) \\
\vdots \\
S_{M}(t, f)
\end{array}\right]
$$

The mixing matrix estimation accuracy is mainly related to the sparsity of signals. For the single source points detection algorithm, any two columns in the mixing matrix should not be correlated and some single source points must exist. If only one source signal $s_{i}$ presets at the time $t$, Formula (6) is rewritten as

$$
\left[\begin{array}{l}
X_{1}(t, f) \\
X_{2}(t, f)
\end{array}\right]=\left[\begin{array}{l}
a_{1 i} \\
a_{2 i}
\end{array}\right] S_{i}(t, f)
$$

For the single source point $\left(t_{p}, f_{p}\right)$ only contain the source signal $s_{1}$, we can obtain

$$
\begin{aligned}
& X_{1}\left(t_{p}, f_{p}\right)=a_{11} S_{1}\left(t_{p}, f_{p}\right)=a_{11}\left\{\operatorname{Re}\left[S_{1}\left(t_{p}, f_{p}\right)\right]+j \operatorname{Im}\left[S_{1}\left(t_{p}, f_{p}\right)\right]\right\} \\
& X_{2}\left(t_{p}, f_{p}\right)=a_{21} S_{1}\left(t_{p}, f_{p}\right)=a_{21}\left\{\operatorname{Re}\left[S_{1}\left(t_{p}, f_{p}\right)\right]+j \operatorname{Im}\left[S_{1}\left(t_{p}, f_{p}\right)\right]\right\}
\end{aligned}
$$

Based on the Equation (8) and the Equation (9), the following formula can be obtained

$$
\begin{gathered}
{\left[\frac{X_{1}\left(t_{p}, f_{p}\right)}{X_{2}\left(t_{p}, f_{p}\right)}\right]^{2}=\frac{a_{11}^{2}\left\{\operatorname{Re}\left[S_{1}\left(t_{p}, f_{p}\right)\right]+j \operatorname{Im}\left[S_{1}\left(t_{p}, f_{p}\right)\right]\right\}^{2}}{a_{21}^{2}\left\{\operatorname{Re}\left[S_{1}\left(t_{p}, f_{p}\right)\right]+j \operatorname{Im}\left[S_{1}\left(t_{p}, f_{p}\right)\right]\right\}^{2}}=\frac{a_{11}^{2}}{a_{21}^{2}}} \\
{\left[\frac{\left|X_{1}\left(t_{p}, f_{p}\right)\right|}{\left|X_{2}\left(t_{p}, f_{p}\right)\right|}\right]^{2}=\left(\frac{\sqrt{a_{11}^{2}\left(\left\{\operatorname{Re}\left[S_{1}\left(t_{p}, f_{p}\right)\right]\right\}^{2}+\left\{\operatorname{Im}\left[S_{1}\left(t_{p}, f_{p}\right)\right]\right\}^{2}\right)}}{\sqrt{a_{21}^{2}\left(\left\{\operatorname{Re}\left[S_{1}\left(t_{p}, f_{p}\right)\right]\right\}^{2}+\left\{\operatorname{Im}\left[S_{1}\left(t_{p}, f_{p}\right)\right]\right\}^{2}\right)}}\right)^{2}=\frac{a_{11}^{2}}{a_{21}^{2}}} \\
{\left[\frac{X_{1}\left(t_{p}, f_{p}\right)}{X_{2}\left(t_{p}, f_{p}\right)}\right]^{2}=\left[\frac{\left.\left|X_{1}\left(t_{p}, f_{p}\right)\right|\right]^{2}}{\left|X_{2}\left(t_{p}, f_{p}\right)\right|}\right]^{2}}
\end{gathered}
$$

If two source signals $s_{1}$ and $s_{2}$ exist at the t-f point $\left(t_{q}, f_{q}\right)$, we can denote the equations as

$$
\begin{aligned}
& X_{1}\left(t_{q}, f_{q}\right)=\left\{a_{11} \operatorname{Re}\left[S_{1}\left(t_{q}, f_{q}\right)\right]+a_{12} \operatorname{Re}\left[S_{2}\left(t_{q}, f_{q}\right]\right\}+j\left\{a_{11} \operatorname{Im}\left[S_{1}\left(t_{q}, f_{q}\right)\right]+a_{12} \operatorname{Im}\left[S_{2}\left(t_{q}, f_{q}\right]\right\}\right.\right. \\
& X_{2}\left(t_{q}, f_{q}\right)=\left\{a_{21} \operatorname{Re}\left[S_{1}\left(t_{q}, f_{q}\right)\right]+a_{22} \operatorname{Re}\left[S_{2}\left(t_{q}, f_{q}\right]\right\}+j\left\{a_{21} \operatorname{Im}\left[S_{1}\left(t_{q}, f_{q}\right)\right]+a_{22} \operatorname{Im}\left[S_{2}\left(t_{q}, f_{q}\right]\right\}\right.\right.
\end{aligned}
$$

The following equations are set

$$
\begin{aligned}
& R_{1}\left(t_{q}, f_{q}\right)=a_{11} \operatorname{Re}\left[S_{1}\left(t_{q}, f_{q}\right)\right]+a_{12} \operatorname{Re}\left[S_{2}\left(t_{q}, f_{q}\right)\right] \\
& I_{1}\left(t_{q}, f_{q}\right)=a_{11} \operatorname{Im}\left[S_{1}\left(t_{q}, f_{q}\right)\right]+a_{12} \operatorname{Im}\left[S_{2}\left(t_{q}, f_{q}\right)\right] \\
& R_{2}\left(t_{q}, f_{q}\right)=a_{21} \operatorname{Re}\left[S_{1}\left(t_{q}, f_{q}\right)\right]+a_{22} \operatorname{Re}\left[S_{2}\left(t_{q}, f_{q}\right)\right]
\end{aligned}
$$




$$
I_{2}\left(t_{q}, f_{q}\right)=a_{21} \operatorname{Im}\left[S_{1}\left(t_{q}, f_{q}\right)\right]+a_{22} \operatorname{Im}\left[S_{2}\left(t_{q}, f_{q}\right)\right]
$$

The Equation (13) and the Equation (14) can also be denoted as

$$
\begin{aligned}
& X_{1}\left(t_{q}, f_{q}\right)=R_{1}\left(t_{q}, f_{q}\right)+j I_{1}\left(t_{q}, f_{q}\right) \\
& X_{2}\left(t_{q}, f_{q}\right)=R_{2}\left(t_{q}, f_{q}\right)+j I_{2}\left(t_{q}, f_{q}\right)
\end{aligned}
$$

Based on the Equation (19) and the Equation (20), the following equations can be obtained

$$
\begin{aligned}
& {\left[\frac{X_{1}\left(t_{q}, f_{q}\right)}{X_{2}\left(t_{q}, f_{q}\right)}\right]^{2}=\frac{\left\{\left[R_{1}\left(t_{q}, f_{q}\right)\right]+j\left[I_{1}\left(t_{q}, f_{q}\right)\right]\right\}^{2}}{\left\{\left[R_{2}\left(t_{q}, f_{q}\right)\right]+j\left[I_{2}\left(t_{q}, f_{q}\right)\right]\right\}^{2}}} \\
& {\left[\frac{\left|X_{1}\left(t_{q}, f_{q}\right)\right|}{\left|X_{2}\left(t_{q}, f_{q}\right)\right|}\right]^{2}=\frac{\left[R_{1}\left(t_{q}, f_{q}\right)\right]^{2}+\left[I_{1}\left(t_{q}, f_{q}\right)\right]^{2}}{\left[R_{2}\left(t_{q}, f_{q}\right)\right]^{2}+\left[I_{2}\left(t_{q}, f_{q}\right)\right]^{2}}}
\end{aligned}
$$

In order to make the Equation (21) to be equal to the Equation (22), the following equation needs to be satisfied

$$
\frac{R_{1}\left(t_{q}, f_{q}\right)}{R_{2}\left(t_{q}, f_{q}\right)}=\frac{I_{1}\left(t_{q}, f_{q}\right)}{I_{2}\left(t_{q}, f_{q}\right)}
$$

The Equation (23) can also be denoted as

$$
\frac{a_{11} \operatorname{Re}\left[S_{1}\left(t_{q}, f_{q}\right)\right]+a_{12} \operatorname{Re}\left[S_{2}\left(t_{q}, f_{q}\right)\right]}{a_{21} \operatorname{Re}\left[S_{1}\left(t_{q}, f_{q}\right)\right]+a_{22} \operatorname{Re}\left[S_{2}\left(t_{q}, f_{q}\right)\right]}=\frac{a_{11} \operatorname{Im}\left[S_{1}\left(t_{q}, f_{q}\right)\right]+a_{12} \operatorname{Im}\left[S_{2}\left(t_{q}, f_{q}\right)\right]}{a_{21} \operatorname{Im}\left[S_{1}\left(t_{q}, f_{q}\right)\right]+a_{22} \operatorname{Im}\left[S_{2}\left(t_{q}, f_{q}\right)\right]}
$$

The above equation is a condition. Based on this condition, the simpler condition can be denoted as

$$
a_{11} a_{22}=a_{12} a_{21}
$$

This condition doesn't satisfy the hypothesis that any two columns are not correlated in the mixing matrix, so the Formula (21) can't be equal to the Formula (22). Similarly, if more source signals exist at some t-f points, the result is similar. For detecting the single source points, we will take advantage of the following formula.

$$
\left[\frac{X_{1}(t, f)}{X_{2}(t, f)}\right]^{2}-\left[\frac{\left|X_{1}(t, f)\right|}{\left|X_{2}(t, f)\right|}\right]^{2}=0
$$

In practice, the above condition is so strict that it is difficult to achieve it. To detect the single source points better, we relax Equation (26) as

$$
\left[\frac{X_{1}(t, f)}{X_{2}(t, f)}\right]^{2}-\left[\frac{\left|X_{1}(t, f)\right|}{\left|X_{2}(t, f)\right|}\right]^{2}<\varepsilon_{1}
$$

Where $\varepsilon_{1}$ is a positive value that is close to 0 , through the above formula, we can detect the single source points.

\section{Mixing Matrix Estimation based on the Improved Potential Function}

To get the estimated mixing matrix, clustering algorithms are usually employed. If observed mixed signals are sparse enough, observed mixed signals have the linear clustering property which is determined by the mixing matrix column vector. When the observed signals are incomplete sparsity, the linear clustering property is not obvious. T-f transform is used to get more obvious linear clustering property. Get the clustering centers by using the clustering of the single source 
points. Then, an estimated mixing matrix can be achieved. In our work, we take advantage of the potential function to obtain the quantity of source signals, then the clustering centers are estimated by the point density theory. First, get the quantity of source signals and achieve a rough estimation of the clustering centers by utilizing the clustering method based on the potential function. The clustering centers are some initial values. More accurate clustering centers need subsequent processing. The improved potential function that makes a rough estimation of the clustering centers can be denoted as

$$
J(z)=\sum_{j=1}^{Q}\left[\exp \left(\frac{\sqrt{x_{j} z^{T}}}{\sigma^{2}}\right)\right]^{\gamma}
$$

in which, $x_{j}$ represents the M-dimensional vector and it belongs to a data set $D=\left\{x_{1}, x_{2}, \cdots, x_{Q}\right\}$ that includes the real part of each single source point. $Q$ denotes the single source point number. $\gamma$ is a parameter that has an impact on estimating the number of peaks and can be estimated with a correlation method. The formula of $\sigma^{2}$ is as follows

$$
\sigma^{2}=\sum_{j=1}^{Q}\left\|x_{j}-\bar{x}\right\|^{2} / Q
$$

Where $\bar{x}=\sum_{j=1}^{Q} x_{j} / Q$. We can change the value of $z$ to get the local maximum of $J(z)$, which can be obtained when $x_{j}$ is equal to $z$. For eliminating some false peaks produced by noises and interferences, the following equation is adopted

$$
\frac{J\left(z_{i}\right)}{\max J\left(z_{i}\right)} \geq \varepsilon_{2}, i=1,2, \cdots, Q
$$

Where $\varepsilon_{2}$ denotes a threshold value and $z_{i}$ represents the clustering center before optimization. This formula is used to remove those small maxima. The number of peaks can be estimated through the peak number produced by the potential function. Meanwhile, the rough estimation of the clustering centers can be obtained through the above-mentioned potential function method.

Then, the distance from one point to another is used to subdivide the single source points. This approach divides these points by introducing the idea of density. If a point is surrounded by many other points, this point is considered to own high density. In this paper, we keep the points with high density and remove the points with low density in order to correct the clustering centers. After getting rid of outliers, the initial clustering centers can be corrected and the new clustering centers will be obtained. Finally, we will get the estimated mixing matrix.

The improved algorithm main steps are as follows:

Step 1 Calculate the $t-f$ coefficients of observed signals through STFT.

Step 2 The new detection strategy is used to detect single source points.

Step 3 Obtain the initial clustering centers by using the potential function-based method.

Step 4 Estimate more accurate clustering centers and finally obtain the mixing matrix.

\section{Simulation Results and Analysis}

To evaluate the performance of our introduced method, four source signals and two observation signals are used for simulation experiments. Four source signals are speech signals described in [21]. Table 1 shows the parameters of specific simulations.

Table 1. Parameters of simulations

\begin{tabular}{cccccc}
\hline Sampling number & STFT size & Overlapping & Weighting function & $\varepsilon_{1}$ & $\varepsilon_{2}$ \\
\hline 160000 & 1024 & 896 & Hanning window & 0.0001 & 0.01 \\
\hline
\end{tabular}


The mixing matrix $A$ is set as

$$
A=\left[\begin{array}{llll}
0.9920 & 0.8499 & 0.5317 & 0.1673 \\
0.1247 & 0.5177 & 0.8472 & 0.9850
\end{array}\right]
$$

STFT is applied to process the observed signals in order to obtain better estimation accuracy. Then, the single source points could be approximated through the single source detection. When there is no noise, the scatter plot of two mixed signals is considered. Because of a large number of t-f points will lead to high computational complexity, it is necessary to reduce some points closing to the origin. Figure 1 displays the scatter plot of two observed signals in t-f plane before single source points detection.

From Figure 1, there are some obvious outliers. Clustering the source points directly can lead to poor estimation performance. Before clustering, abnormal values should be eliminated to get better performance. So, the detection process of single source points is necessary. In addition, it can be seen that there are some points in the vicinity of the origin in Figure 1. These points have much less effect on estimating the directions of the lines than those that are away from the origin, so better linear clustering property and lower computational complexity can be obtained after removing these points. After doing single source point detection and removing the points near the origin, we can get the scatter plot in Figure 2.

As shown in Figure 2, some outliers have been removed by single source detection.

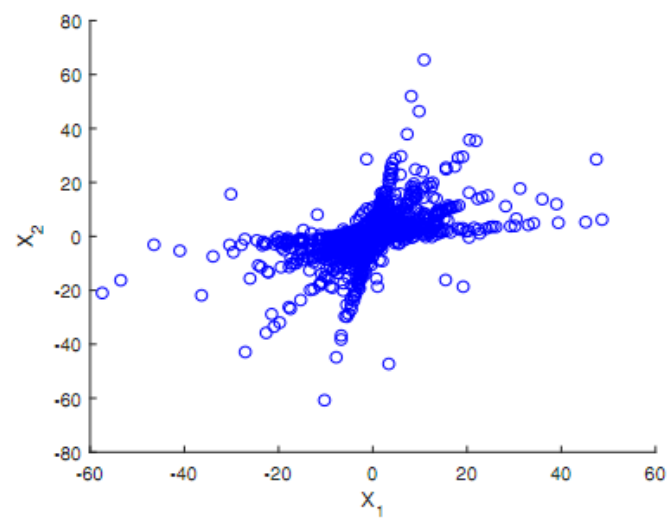

Figure 1. The scatter plot of two observed signals in t-f plane before detecting single source points

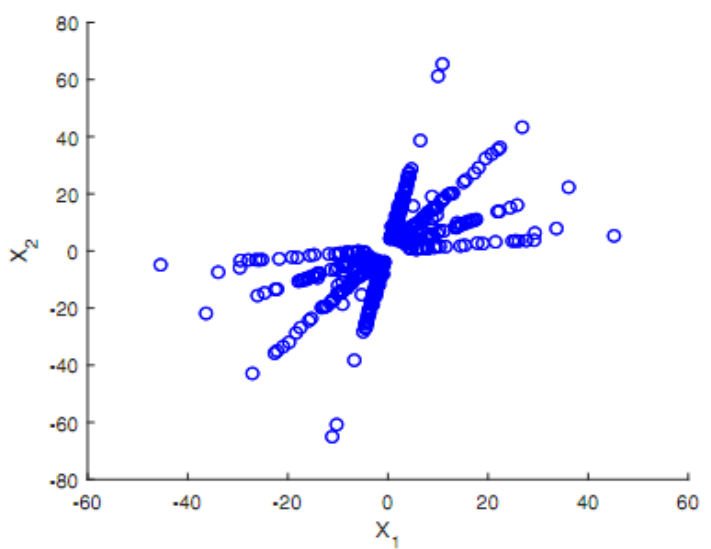

Figure 2. The scatter plot of two observed signals in t-f plane after single source point detection and removing the points near the origin

The scatter plot of two observed signals in t-f plane with the sign changes and normalization can be shown in Figure 3.

In Figure 3, these points have the clustering property but there are still some interferences. The potential function is introduced to process these points. Figure 4 shows the result processed by the potential function. 


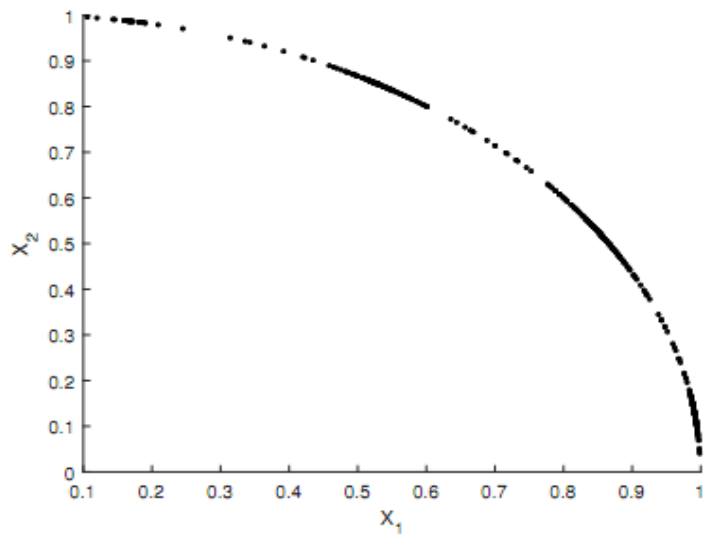

Figure 3. The scatter plot of two observed signals in $t-f$ domain with the sign changes and normalization

From Figure 4, the number of peaks corresponds to the quantity of the source signals. Meanwhile, the positions of peaks are the estimated values of the mixing matrix. The initial clustering centers can help us get the mixing matrix, but the result is not satisfactory. A more accurate clustering result is needed, and the point density theory is adopted. Based on the density distribution of the points, some points are removed in order to get better performance. The two observed signals scatter plot in t-f plane with the sign changes and normalization after removing some points with low density are shown in Figure 5 .

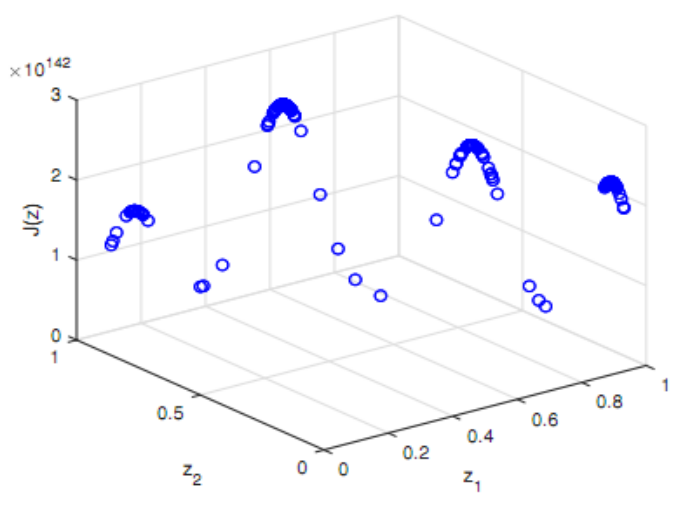

Figure 4. Three-dimensional plot of $J(z)$

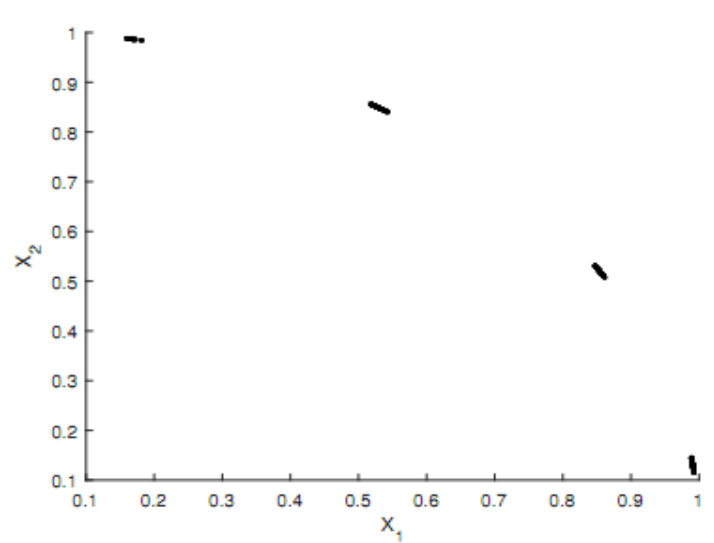

Figure 5. The scatter plot of two observed signals in t-f plane with the sign changes and normalization after removing some points with low density

As shown in Figure 5, a better clustering property is present after removing the points with low density. The corrected clustering centers are estimated based on the point density theory. And finally we can obtain the estimated mixing matrix 


$$
\tilde{A}=\left[\begin{array}{llll}
0.9915 & 0.8509 & 0.5320 & 0.1700 \\
0.1296 & 0.5206 & 0.8468 & 0.9850
\end{array}\right]
$$

Comparing the estimated mixing matrix with the original one, estimated values are close to the real values. It is illustrated that our studied algorithm can estimate the mixing matrix well. For the sake of illustrating the universality of the proposed algorithm, we carried out another simulation to test the proposed algorithm. Other conditions remain changeless. After the experiment, the original mixing matrix and the estimated one are as shown

$$
\begin{aligned}
A & =\left[\begin{array}{llll}
0.5162 & 0.2015 & 0.7618 & 0.9905 \\
0.8569 & 0.9763 & 0.6477 & 0.1377
\end{array}\right] \\
\tilde{A} & =\left[\begin{array}{llll}
0.5133 & 0.2047 & 0.7628 & 0.9904 \\
0.8584 & 0.9772 & 0.6466 & 0.1385
\end{array}\right]
\end{aligned}
$$

The above experiment proves that the above algorithm is able to estimate other mixing matrices effectively.

For determining the effectiveness of our studied algorithm, we make use of the normalized mean square error (NMSE) to evaluate the estimated mixing matrix, whose definition is

$$
N M S E=10 \log \left(\frac{\sum_{i, j}\left(\tilde{a}_{i j}-a_{i j}\right)^{2}}{\sum_{i, j} a_{i, j}^{2}}\right)
$$

Where $\tilde{a}_{i j}$ and $a_{i j}$ represent the $(i, j)^{\text {th }}$ element of the estimated mixing matrix and the $(i, j)^{\text {th }}$ element of the real one, respectively. Our proposed algorithm is compared with the other algorithms in Literature [21] or [22]. In a noisy case, the relative robustness of the algorithm is evaluated by adding Gaussian white noises. The curves of average NMSE for each algorithm obtained by 100 Monte Carlo trials are displayed in Figure 6.

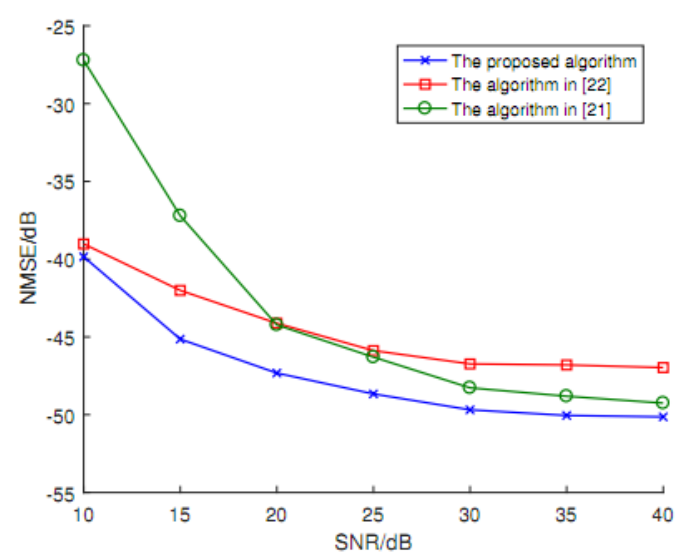

Figure 6. The comparison of the proposed algorithm and other algorithms

As shown in Figure 6, our proposed algorithm is more robust when noises are present. The proposed algorithm is able to estimate the mixing matrix more accurately compared with other comparison algorithms.

\section{Conclusions}

An improved mixing matrix estimation algorithm was built in this paper as the number of the source signals is unknown, this method can estimate the mixing matrix more accurately and stably. A new single source point detection rule is proposed in t-f plane to get better sparsity of the observed signals. The clustering algorithm based on potential function was used to obtain the estimated value of the source signal number and initial clustering centers. (rewrite). This algorithm solves the problems that the clustering results are unstable, and the accuracy is low. 


\section{Acknowledgements}

The work is funded by the National key research and development program (Grant No. 2016YFF0102806).

\section{References}

1. J. J. Yang and H. L. Liu, "Blind Identification of the Underdetermined Mixing Matrix based on K-Weighted Hyperline Clustering," Neurocomputing, Vol. 149, No. PB, pp. 483-489, 2015

2. M. T. Huang, C. H. Lee, and C. M. Lin, "Blind Source Separation with Adaptive Learning Rates for Image Encryption," Journal of Intelligent and Fuzzy Systems, Vol. 30, No. 1, pp. 451-460, 2015

3. J. Liu, S. E. Yang, S. C. Piao, and Y. W. Huang, "Blind Source Separation of Ship-Radiated Noise using Single Observing Channel," ACTA ACUSTICA, Vol. 36, No. 3, pp. 265-270, 2011

4. Z. C. Shan, C. S. Lin, and Q. Xiang, "The Separation of Ship Signal based on Second Order Nonstationary Statistic," SIGNAL PROCESSING, Vol. 25, No. 6, pp. 973-976, 2009

5. F. Andreotti, J. Behar, S. Zaunseder, J. Oster, and G. D. Clifford, "An Open-Source Framework for Stress-Testing NonInvasive Foetal ECG Extraction Algorithms,” Physiological Measurement, Vol. 37, No. 5, pp. 627-648, 2016

6. S. Farashi, "Spike Detection using a Multiresolution Entropy based Method," Biomedical Engineering-Biomedizinische Technik, Vol. 63, No. 4, pp. 361-376, 2018

7. M. G. S. Sriyananda, J. Joutsensalo, and T. Hamalainen, "Blind Source Separation based Interference Suppression Schemes for OFDM and DS-CDMA,” Telecommunication Systems, Vol. 4, No. 2, pp. 1-10, 2016

8. Z. C. Sha, Z. T. Huang, Y. Y. Zhou, and F. H. Wang, "Frequency-Hopping Signals Sorting based on Underdetermined Blind Source Separation," IET Communications, Vol. 7, No. 14, pp. 1456-1464, 2013

9. G. R. Naik and D. K. Kumar, "An Overview of Independent Component Analysis and its Applications," International Journal of Computing and Information Sciences, Vol. 35, No. 1, pp. 63-81, 2011

10. X. He, F. He, and T. Zhu, "Large-Scale Super-Gaussian Sources Separation using Fast-ICA with Rational Nonlinearities," International Journal of Adaptive Control and Signal Processing, Vol. 31, No. 3, pp. 379-397, 2017

11. S. Qin, J. Guo, and C. Zhu, "Sparse Component Analysis using Time-Frequency Representations for Operational Modal Analysis," Sensors, Vol. 15, No. 3, pp. 6497-6519, 2015

12. Y. Zhong, X. Wang, L. Zhao, R. Y. Feng, L. P. Zhang, and Y. Y. Xu, "Blind Spectral Unmixing based on Sparse Component Analysis for Hyperspectral Remote Sensing Imagery," Isprs Journal of Photogrammetry and Remote Sensing, Vol. 119, pp. 4963, 2016

13. Y. Li, S. I. Amari, A. Cichocki, D. W. C. Ho, and S. L. Xie, "Underdetermined Blind Source Separation based on Sparse Representation," IEEE Transactions on Signal Processing, Vol. 54, No. 2, pp. 423-437, 2006

14. O. Yilmaz and S. Rickard, "Blind Separation of Speech Mixtures Via Time-Frequency Masking," IEEE Transactions on Signal Processing, Vol. 52, No. 7, pp. 1830-1847, 2004

15. A. Aissa-El-Bey, N. Linh-Trung, K. Abed-Meraim, A. Belouchrani, and Y. Grenier, "Underdetermined Blind Separation of Nondisjoint Sources in the Time-Frequency Domain," IEEE Transactions on Signal Processing, Vol. 55, No. 3, pp. 897-907, 2007

16. J. Thiagarajan, K. Natesan Ramamurthy, and A. Spanias, "Mixing Matrix Estimation using Discriminative Clustering for Blind Source Separation," Digital Signal Processing, Vol. 23, No. 1, pp. 9-18, 2013

17. F. C. Feng and M. Kowalski, "Revisiting Sparse ICA from a Synthesis Point of View: Blind Source Separation for over and Underdetermined Mixtures," Signal Processing, Vol. 152, pp. 165-177, 2018

18. F. Abrard and Y. Deville, "A Time-Frequency Blind Signal Separation Method Applicable to Underdetermined Mixtures of Dependent Sources," Signal Processing, Vol. 85, No. 7, pp. 1389-1403, 2005

19. Q. Guo and G. Q. Ruan, "A Complex-Valued Mixing Matrix Estimation Algorithm for Underdetermined Blind Source Separation," Circuits Systems and Signal Processing, Vol. 37, No. 8, pp. 3206-3226, 2018

20. F. Lu, Z. Huang, and W. Jiang, "Underdetermined Blind Separation of Non-Disjoint Signals in Time-Frequency Domain based on Matrix Diagonalization," Signal Processing, Vol. 91, No. 7, pp. 1568-1577, 2011

21. V. G. Reju, S. N. Koh, and I. Y. Soon, "An Algorithm for Mixing Matrix Estimation in Instantaneous Blind Source Separation," Signal Processing, Vol. 89, No. 9, pp. 1762-1773, 2009

22. T. Dong, Y. Lei, and J. Yang, “An Algorithm for Underdetermined Mixing Matrix Estimation,” Neurocomputing, Vol. 104, pp. 26-34, 2013 